\title{
Nasonia tools up
}

Evolutionary developmental biology (evo-devo) research has been boosted in recent years by expansion in the range of genetically tractable model organisms that can be manipulated in the laboratory. The publication of the genome sequence of three species of the parasitoid wasp Nasonia - accompanied by a clutch of papers that have utilized the sequence data - is enabling the development of a sophisticated genetic tool kit for this emerging arthropod model. This work opens new avenues in evo-devo research, as well having potential benefits for biological pest control.

The Nasonia Genome Working Group sequenced and annotated the genome of a highly inbred line of Nasonia vitripennis by Sanger sequencing. They then used this as a reference to build the genomes of two closely related species - Nasonia giraulti and Nasonia longicornis from a combination of Sanger and short-read sequencing data. They found that the repertoire of genes in these wasps is fairly typical of insects but, unlike the classical insect model Drosophila melanogaster, Nasonia species have a full complement of DNA methylation genes. They also have a large set of venom genes, and because these have a higher degree of divergence among Nasonia species than non-venom genes, they might be important for adaptation to different hosts, and could also be valuable for drug development.

The appeal of Nasonia species as model organisms stems from their short generation time, haploid male genetics and - when cured of infection with the intracellular Wolbachia bacteria - cross-species fertility. The genome sequence now expands the available resources and tools. For example, Desjardins and colleagues introgressed a locus that confers preference for different types of flies as hosts among Nasonia species and mapped it using a new genotyping array. Understanding

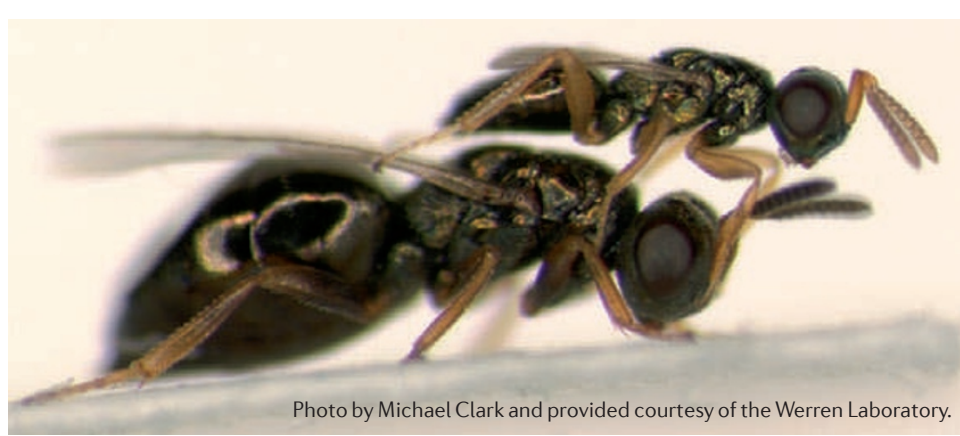

how morphological differences among species evolve at a genetic level is central to evo-devo research, and Loehlin et al. showed that an interspecies QTL for a morphological trait - wing size - could be efficiently identified and cloned in Nasonia species. The QTL implicates the sex-determining gene doublesex in the recent evolution of sex-specific differences. The genome sequence has also aided characterization of the evolution of a newly identified species, Nasonia oneida.

The potential for fine-scale mapping and positional cloning of QTLs is likely to enable researchers to make substantial advances in understanding the genetic basis of arthropod developmental evolution and host-parasite interactions. Further knowledge of Nasonia biology could also improve the way in which they are used to control the agricultural pests they parasitize.

Mary Muers

\section{ORIGINAL RESEARCH PAPERS}

Nasonia Genome Working Group. Functional and evolutionary insights from the genomes of three parasitoid Nasonia species. Science 327, 343-348 (2010)|Desjardins, C. A., Perfectti, F., Bartos, J. D., Enders, L. S. \& Werren, J. H. The genetic basis of interspecies host preference differences in the model parasitoid Nasonia. Heredity 20 Jan 2010 (doi:10.1038/hdy.2009.125) | Loehlin, D. W. et al. Non-coding changes cause sex-specific wing size differences between closely related species of Nasonia. PLoS Genet. 6, e1000821 (2010) FURTHER READING Sommer, R. J. The future of evo-devo: model systems and evolutionary theory. Nature Rev. Genet. 10, 416-422 (2009) 